\title{
MATLAB Simulation Research on Static Var Compensator
}

\author{
JinBo Chen ${ }^{1,2, a}$, WenYu Hu${ }^{1,2, b^{*}}$ \\ ${ }^{1}$ School of Computer Science and Mathematics, Fujian University of Technology, Fuzhou, Fujian, 350118, China \\ ${ }^{2}$ Fujian Provincial Key Laboratory of Big Data Mining and Applications (Fujian University of Technology), Fuzhou, Fujian, 350118, China
}

\begin{abstract}
TCR-TSC static reactive power compensator (SVC) is the most widely used in the field of power system reactive power compensation. This type of reactive power compensator can not only compensate the reactive power required in the power system, but also handle the over-compensation problem well. This paper will establish a MATLAB simulation model to simulate the TCR-TSC SVC, focusing on the dynamic reactive power compensation characteristics of the TCR-TSC SVC in suppressing voltage fluctuations. The simulation results show that the TCR-TSC SVC has a better dynamic reactive power compensation effect.
\end{abstract}

\section{Introduction}

Parallel SVC is the most common reactive power compensation equipment in the current $\mathrm{AC}$ power grid. It can achieve dynamic and continuous compensation of reactive power, absorb harmonics generated by the power grid and reactive power compensation equipment itself, and suppress voltage fluctuations and voltage flicker ${ }^{[1-2]}$. Early reactive power compensation equipment generally used synchronous phase modifier, but from the perspective of economic operation, the operating costs of synchronous phase modifier reactive power compensation were relatively high. Later, in order to reduce operating costs, a large number of static capacitors were used for reactive power compensation in the power grid. But because the impedance of the static capacitor is fixed, continuous dynamic compensation cannot be achieved. In order to solve this problem, people began to use static reactive power compensation equipment composed of thyristor switching capacitor (TSC) branch and thyristor control reactor (TCR) in parallel ${ }^{[3]}$.

The second part of this article introduces the research related to this article; the third part builds a TCR-TSC static var compensator MATLAB simulation model; the fourth part analyzes the simulation results; finally, the full text is summarized.

\section{Related work}

Literature [4] studied the coupling and interaction between SVC and HVDC transmission system. Literature [5] pointed out that SVC has an important influence on the stability of wind power grid-connected system through eigenvalue analysis and simulation analysis. Literature [6] Using eigenvalue analysis method or impedance analysis method to study the influence of static var generator (SVG) on subsynchronous oscillation of wind power gridconnected system.

\section{MATLAB simulation model}

\subsection{The basic structure of TSC-TCR SVC}

The simple schematic diagram of the TSC-TCR SVC is shown in Figure 1. There are three TSC branches and one TCR branch in the figure. The TSC-TCR SVC is installed on the low-voltage side of the step-down transformer, and the filter is mainly used to filter out the harmonic current generated by the SVC device. The TCR branch is composed of a reactor and a pair of anti-parallel thyristors in series, and the TSC branch is composed of a capacitor and a pair of anti-parallel thyristors in series. 


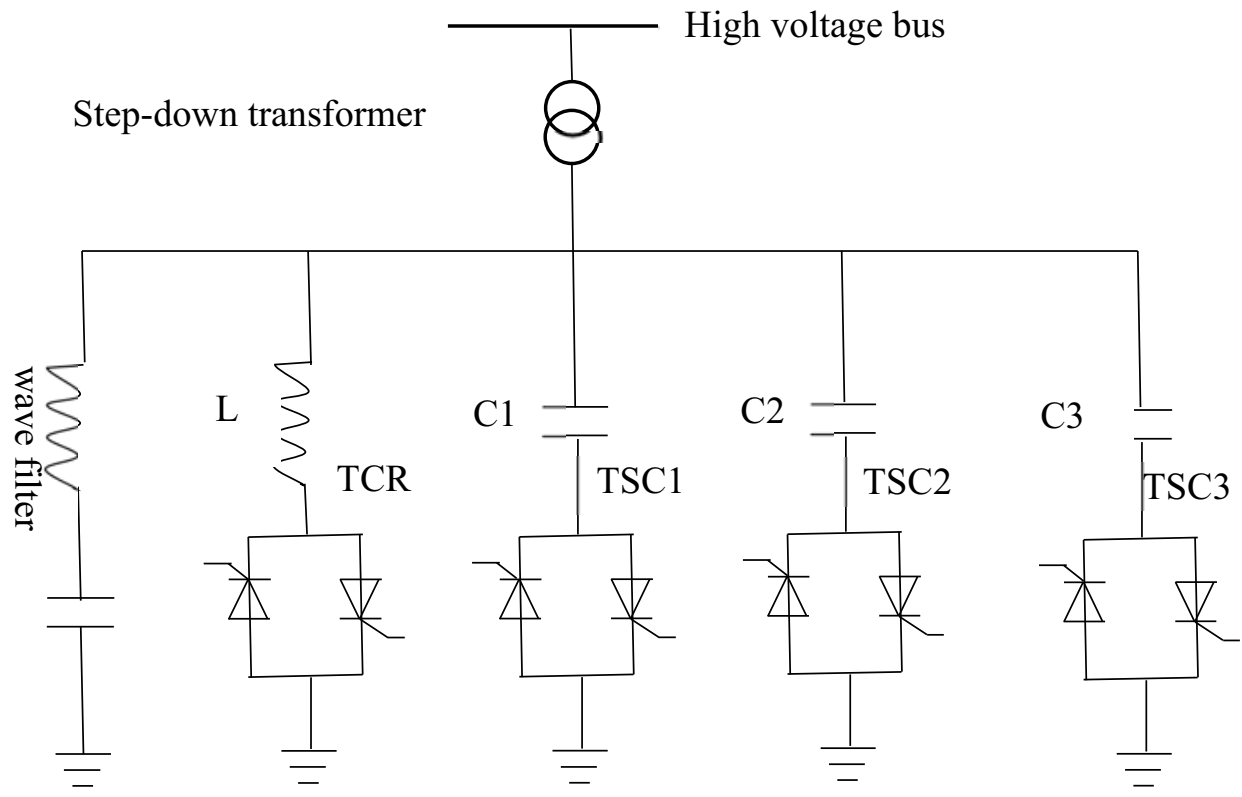

Figure 1. Basic structure of SVC

In the actual SVC device, it is generally composed of one TCR branch and multiple TSC branches, but in order to make the reactive power output by the SVC device both capacitive and inductive, generally speaking, the capacity of a single TCR branch It is greater than the capacity of a single TSC branch. Since the reactive power compensated by the TSC branch is discontinuous, over-compensation often occurs. The parallel TCR branch can solve the problem of over-compensation and realize the continuous adjustment of the reactive power.

\subsection{Control principle of TSC-TCR SVC}

Figure 2 shows the control principle diagram of the TSCTCR SVC. The primary and secondary voltages measured by the V-I measurement module at both ends of the transformer are sent to the SVC controller. The SVC controller is mainly composed of four parts: measurement subsystem, voltage regulation system, distribution unit, and trigger unit. The primary side voltage is sent to the measurement subsystem to obtain the measured voltage, and the difference between the measured voltage and the reference voltage is sent to the voltage regulation system to obtain the susceptance value required for reactive power compensation. The susceptance value is sent to the distribution unit, and the distribution unit determines the required trigger angle of the TCR branch thyristor and the number of TSC branches. Then the trigger unit sends a trigger pulse according to the trigger angle of the TCR branch thyristor and the number of TSC branches obtained by the allocation unit to control the on and off of the TCR branch and the switching of the TSC branch. The capacitance of the TSC branch is controlled by switching, and the inductance is controlled by controlling the firing angle of the thyristor for the TCR branch. When the voltage of the power system is low, the capacitive reactive power is compensated by inputting the TSC branch. When the capacitive reactive power input is too much, it is necessary to input the TCR branch to absorb the excess capacitive reactive power. 


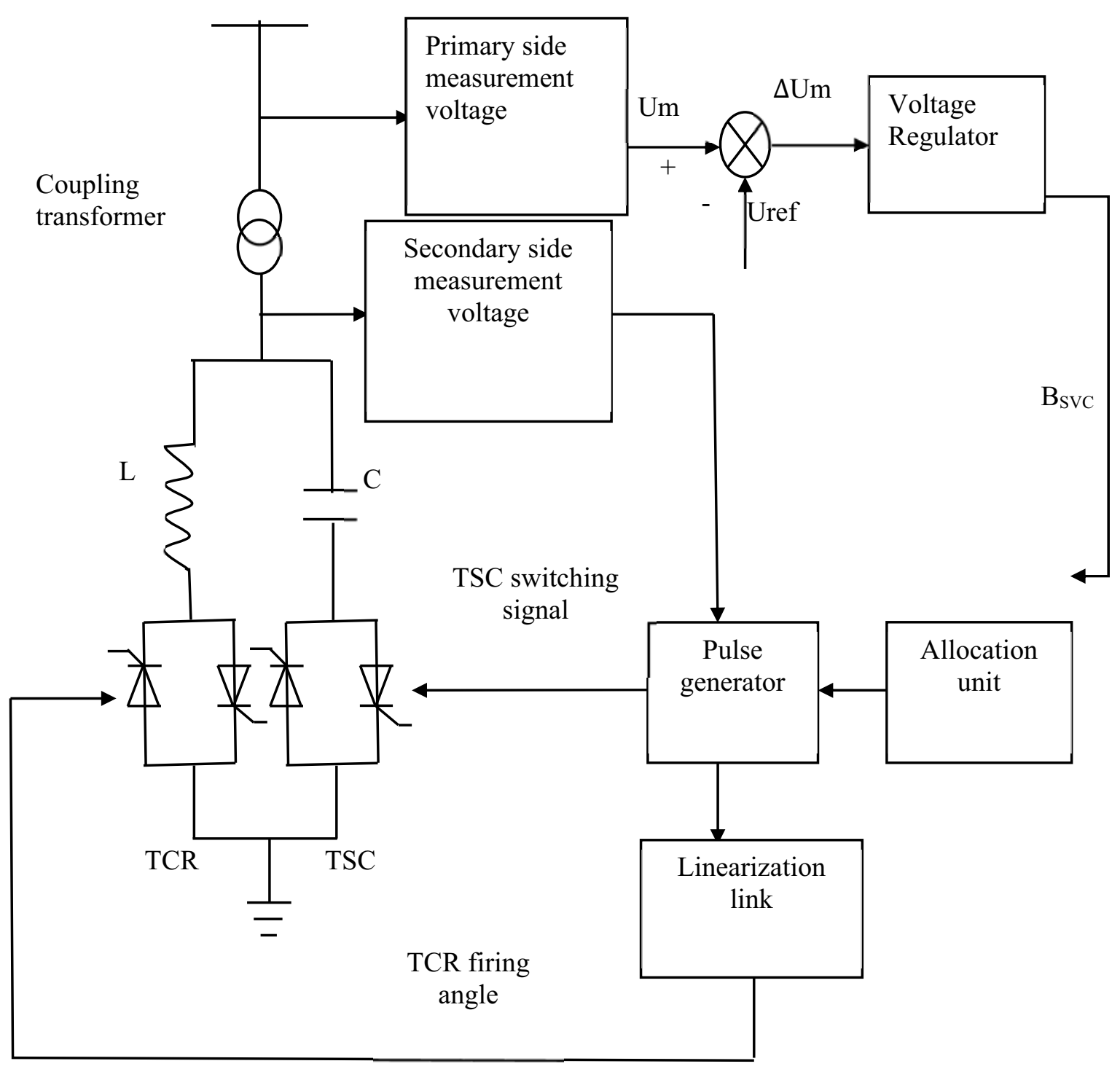

Figure 2. Control principle diagram of TSC-TCR SVC

\subsection{MATLAB simulation model of TSC-TCR SVC}

According to the basic structure diagram and control principle diagram of TSC-TCR SVC shown above, a MATLAB simulation model is built. The simulation model shown in Figure 3: 


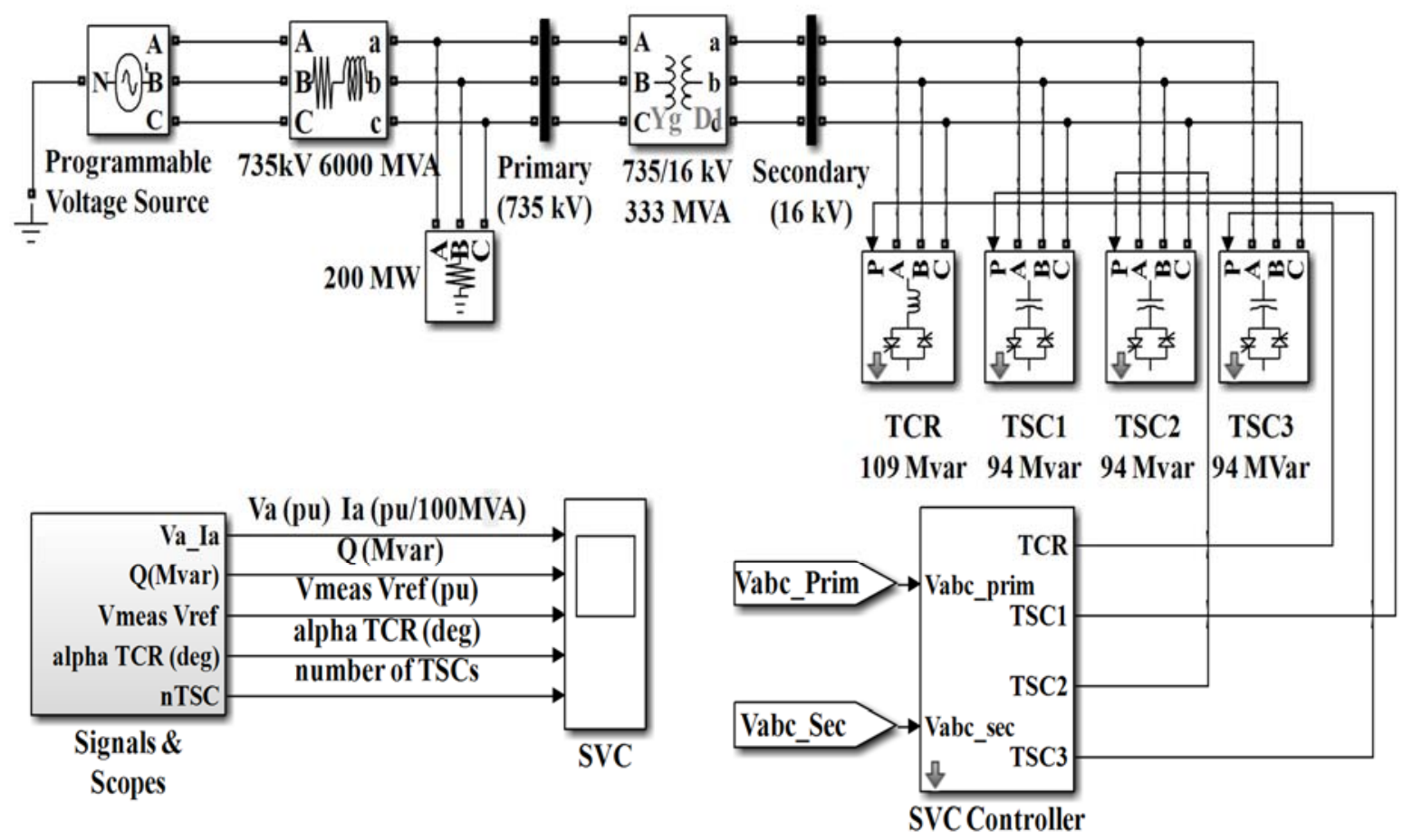

Figure 3. MATLAB simulation model of TSC-TCR SVC

\section{Simulation results and analysis}

This simulation is mainly carried out around the goal of maintaining the voltage stability of the SVC, and then studying the dynamic characteristics of the SVC. The simulation model consists of a 6000MVA resistance and inductance power supply branch, a 200MW load, a transformer with a transformation ratio of $735 / 16 \mathrm{KV}$ and a capacity of 333MVA, three TSC branches with a capacity of $94 \mathrm{Mvar}$, and a TCR branch with a capacity of 109Mvar. At the same time, the programmable voltage source is selected as the equivalent power supply of the simulation model, the reference voltage is set to $1.0 \mathrm{pu}$, and the three time nodes of $0.1 \mathrm{~s}, 0.4 \mathrm{~s}$, and $0.7 \mathrm{~s}$ are set as the time points of the voltage change. The voltage amplitudes corresponding to the three time points are $1.025 \mathrm{pu}$, $0.934 \mathrm{pu}$, and $1.0 \mathrm{pu}$, respectively.

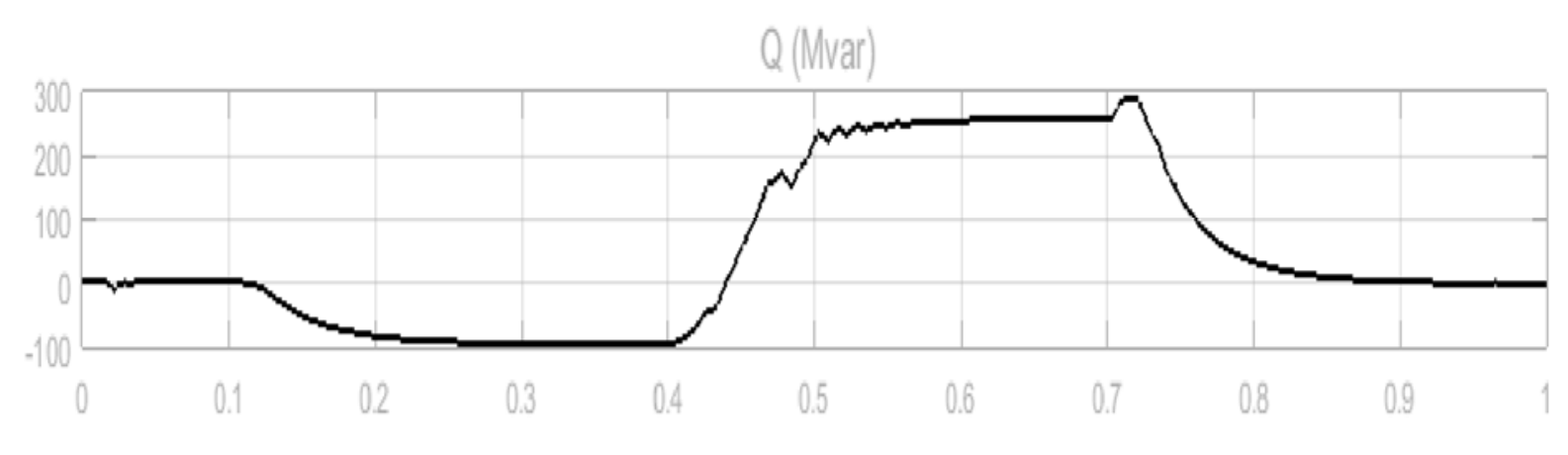

Figure 4. Reactive power output by SVC 


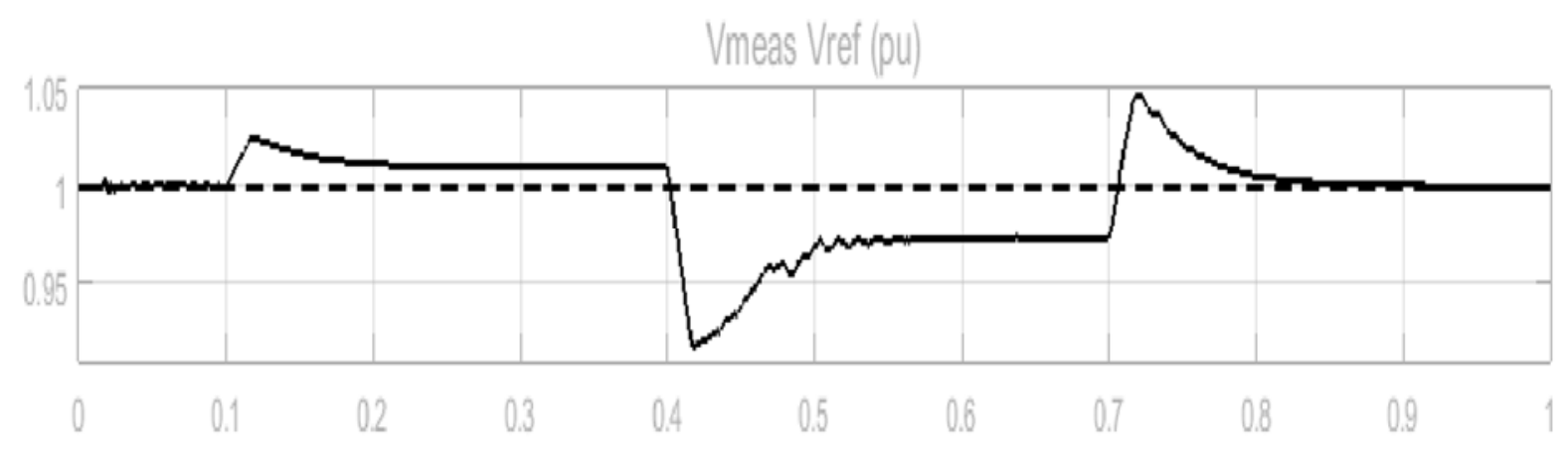

Figure 5. Measuring voltage, reference voltage

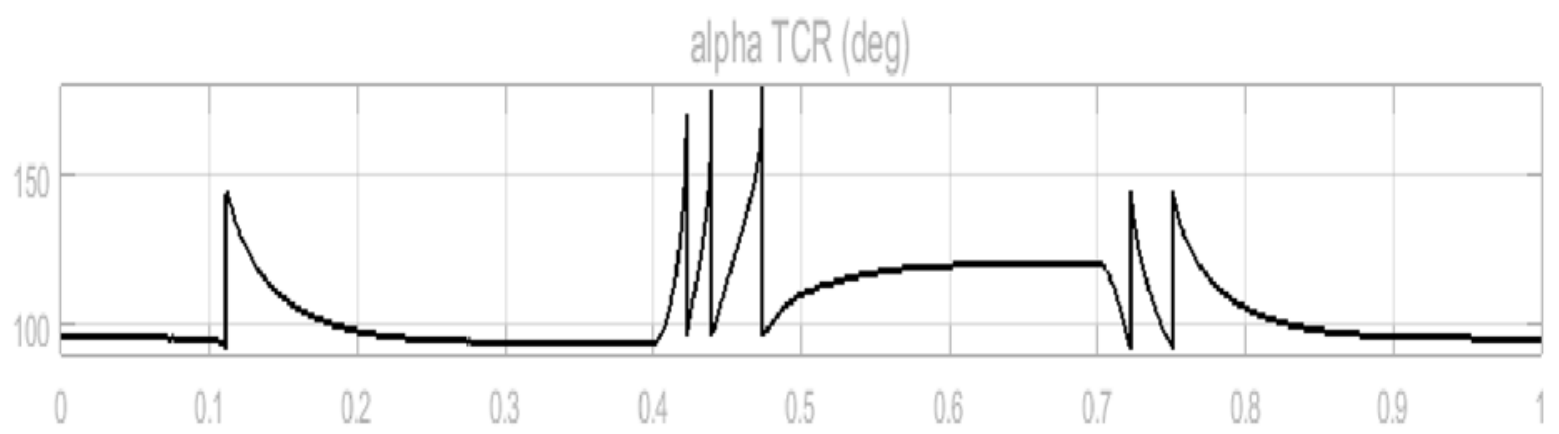

Figure 6. Trigger angle of thyristor

The voltage of the programmable voltage source is set to $1.004 \mathrm{pu}$. The delayed firing angle of the thyristor of the TCR branch is $\alpha=96^{\circ}$, and the measured voltage is $1 \mathrm{pu}$, which does not require reactive power compensation. When $\mathrm{t}=0.1 \mathrm{~s}$, the power supply voltage increases to $1.025 \mathrm{pu}$. At this time, SVC will absorb 95 Mvar of reactive power in order to restore the voltage to the reference voltage. The delay firing angle of the thyristor of the TCR branch is $\alpha=94^{\circ}$, and the time required for the measured voltage to return to $1.01 \mathrm{pu}$ is about $0.135 \mathrm{~s}$.

When $\mathrm{t}=0.4 \mathrm{~s}$, the power supply voltage drops to 0.934 pu. At this time, SVC will emit 256 Mvar reactive power in order to restore the voltage to the reference voltage. TCR absorbs about $40 \%$ of the reactive power, and the delay firing angle of the thyristor of the TCR branch is $\alpha=120^{\circ}$.

When $\mathrm{t}=1.0 \mathrm{~s}$, the power supply voltage rises to $1.0 \mathrm{pu}$. At this time, SVC no longer plays a role, neither absorbing reactive power nor emitting reactive power.

\section{Conclusion}

SVC can maintain the voltage of the power system within $\pm 10 \%$ of the reference voltage, which has a significant effect on improving the voltage stability of the power system. At the same time, it also plays a role in increasing the power factor, reducing losses, increasing the transmission capacity of the grid, and improving the quality of user voltage. Today, when the harmonic pollution in the power system is becoming increasingly severe, the application of SVC can effectively suppress the harmonics.

\section{Acknowledgments}

This article is one of the phased achievements of the National Key R\&D Program of the Ministry of Science and Technology(2018YFC1201103)

\section{References}

1. GE W C. (2018) Voltage stability and dynamic reactive power compensation of power- system. Science Press, Beijing.

2. CHENG H X. (2016) Theory and application of reactive power compensation. China Machine Press, Beijing.

3. MOHANMATHUR R, VARMA R K. (2002) Thyristor-based FACTS controllers for electrical transmission systems. Wiley-IEEE Press, Plano, TX.

4. SZECHTMAN M, F. LONG W, GOLE A, et al. (1999) Coordination of controls of multiple FACTS/HVDC links in the same system. CIGRE, Paris.

5. LIU H T, XU Z, ZHOU C C. (2003) A Study on subsynchronous oscillation of generation set connected to static VAR compensator. Power System Technology, 2003(1):1-4.

6. SUN X D, LIU G H, LV X G, et al. (2020) Research and application of subsynchronous oscillation suppression method based on SVG. Power Electronics, 54(3):75-78. 